\title{
ON THE ASYMPTOTIC BEHAVIOUR OF RANDOM RECURSIVE TREES IN RANDOM ENVIRONMENTS
}

\author{
K. A. BOROVKOV,$^{*}$ The University of Melbourne \\ V. A. VATUTIN, ${ }^{* *}$ Steklov Mathematical Institute
}

\begin{abstract}
We consider growing random recursive trees in random environments, in which at each step a new vertex is attached (by an edge of random length) to an existing tree vertex according to a probability distribution that assigns the tree vertices masses proportional to their random weights. The main aim of the paper is to study the asymptotic behaviour of the distance from the newly inserted vertex to the tree's root and that of the mean numbers of outgoing vertices as the number of steps tends to $\infty$. Most of the results are obtained under the assumption that the random weights have a product form with independent, identically distributed factors.
\end{abstract}

Keywords: Random recursive tree; random environment; Spitzer's condition; distance to the root; outdegree

2000 Mathematics Subject Classification: Primary 05C 80

Secondary 60G50; 05C05; 60F99

\section{Introduction}

We consider the following random recursive tree model. A recursive tree is constructed incrementally, by attaching a new vertex to a randomly chosen existing tree vertex at each step. Initially, the tree consists of a single vertex, $v(0)$, that has weight $w(0)=1$ and label 0 . At the first step, a new vertex, $v(1)$, is added to the tree as a child of the initial vertex. It is labelled 1 , and a random weight, $w(1)>0$, and a random length, $Y(1) \geq 0$, are respectively assigned to $v(1)$ and to the edge connecting the vertices $v(0)$ and $v(1)$. It is assumed that the edge is directed from $v(0)$ to $v(1)$. At step $j>1$, given all the weights $w(0), w(1), \ldots, w(j-1)$, first a node $v\left(j^{*}\right)$ is chosen at random from the nodes $v(0), v(1), \ldots, v(j-1)$ according to the distribution with probabilities proportional to the nodes' weights, and then a new vertex $v(j)$ is added to the tree as a child of the node $v\left(j^{*}\right)$. The new vertex has label $j$, and a random weight $w(j)>0$ and a random length $Y(j) \geq 0$ are respectively assigned to it and to the edge connecting the vertices $v\left(j^{*}\right)$ and $v(j)$. As at the initial step (where, for consistency, we will put $1^{*}=0$ ), the edge is directed from $v\left(j^{*}\right)$ to its child vertex $v(j)$. We assume that $\{Y(j)\}_{j \geq 1}$ is a sequence of independent random variables (RVs) which is independent of the sequence of the (generally speaking random) weights $\{w(j)\}_{j \geq 0}$. Interpreting the sequence of weights as a 'random environment' in which our recursive tree is growing, and appealing to an analogy with random walks and branching processes in random environments, it is not unnatural to refer to such a model as a random recursive tree in a random environment.

Received 28 February 2006; revision received 15 September 2006.

* Postal address: Department of Mathematics and Statistics, The University of Melbourne, Parkville, VIC 3010,

Australia. Email address: kostya@ms.unimelb.edu.au

** Postal address: Steklov Mathematical Institute RAS, Gubkin St. 8, 119991 Moscow, Russia. 
Let

$$
D_{0}:=0, \quad D_{n}:=D_{n^{*}}+Y(n), \quad n \geq 1,
$$

be the distance from the vertex $v(n)$ to the root (i.e. the sum of the lengths of the edges connecting $v(n)$ with $v(0))$. Our main aim in this paper is to study, as $n \rightarrow \infty$, the asymptotic behaviour of $D_{n}$ under various assumptions on the random weights $w(j)$ and lengths $Y(j)$, and also that of the mean values of the outgoing degrees

$$
N_{n}(j):=\sum_{k=j+1}^{n} \mathbf{1}_{\left\{v\left(k^{*}\right)=v(j)\right\}}, \quad j \leq n,
$$

where $\mathbf{1}_{A}$ is the indicator of the event $A$.

Observe that if $w(j) \equiv Y(j) \equiv 1$ for all $j$, then we obtain the standard random recursive tree ([11]; see also [15]). If $w(j)=a^{j}, j \geq 0$, where $a>0$ is a constant and $Y(j), j \geq 1$, are RVs whose distributions satisfy certain mild conditions, we obtain the recursive tree considered in [10] (in fact, the model of [10] assumed that at each step a fixed number, $k \geq 1$, of children are attached to one of the existing tree vertices, and also that the $Y(j)$ are vector valued).

Here we should also mention other, related models where the weights of the vertices can change at each step. Thus, if, after the completion of the $k$ th step of the tree construction, the weight of the vertex $v(j), j \leq k$, is $w(j) \equiv w(j, k)=1+\beta N_{k}(j)$, where $\beta \geq 0$ is constant and $Y(j) \equiv 1$, we obtain the linear recursive tree studied in [16] and [5] (see the bibliographies therein for further references). The case where $w(j) \equiv w(j, k)=1+N_{k}(j)$ was considered in [3]; the power-tail limiting behaviour of the degree distribution for this model that had been guessed in [3] was established in [8].

If $w(j)=a_{1} \cdots a_{j}, j \geq 1$ (where $a_{1}, \ldots, a_{j}$ are independent, identically distributed (i.i.d.) $\mathrm{RVs})$, and $Y(j) \equiv 1$, we obtain a version of a weighted recursive tree. It is this last model and its generalizations that will be of the most interest to us in the present paper.

From now on we assume that the weight, $w(j)$, of the vertex $v(j)$ is, generally speaking, random and, once assigned, remains unchanged forever.

Section 2 of the paper is devoted to studying the asymptotic behaviour of the distribution of $D_{n}$. Theorems 1 and 2 present general convergence results for the conditional distribution of $D_{n}$ in the cases where the random weights $w(j)$ tend to 'prescribe' new attachments to vertices close to the root of the tree and, respectively, where the new attachments are 'more dispersed' across the tree. Corollary 2 covers the special case where $w(j) \equiv 1$. The results of Section 2 also show that, for any $\alpha \in(0,1]$, we can construct a random recursive tree such that $D_{n}$ behaves like $n^{\alpha}$ as $n \rightarrow \infty$. Theorem 3 implies that, in the case of the 'productform' weights $w(j)=a_{1} \cdots a_{j}, j \geq 1$, with $a_{j}$ being nondegenerate i.i.d. RVs satisfying the moment conditions $\mathrm{E} \ln a_{j}=0$ and $\mathrm{E}\left|\ln a_{j}\right|^{2+\delta}<\infty$ for some $\delta>0$, the limiting distribution of $D_{n} / \sqrt{n}$ coincides with the law of the maximum of the Brownian motion process on a finite time interval.

Section 3 deals with the expectations of the numbers of outgoing degrees in the case of product-form weights, under the assumption that the random walk generated by the i.i.d. sequence $\left\{\ln a_{j}\right\}$ satisfies Spitzer's condition. Theorem 4 gives the asymptotic behaviour of the unconditional expectations $\mathrm{E} N_{n}(k)$ as $n \rightarrow \infty$ when either $k=j$ or $k=n-j$ for a fixed value $j \geq 0$ (in both cases it is given by a regularly varying function of $n$ ). Theorem 5 complements it by covering the case where $\min \{j, n-j\} \rightarrow \infty$. Here the answer has the form of a product of regularly varying functions of $j$ and $n-j$, respectively; in particular, in the case when $\ln a_{j}$ has zero mean and finite variance, we obtain $\mathrm{E} N_{n}(j) \sim 2 \pi^{-1}(n-j)^{1 / 2} j^{-1 / 2}$. 
Theorem 6 describes, for a range of $j$-values, the asymptotic behaviour of the distribution of the conditional expectation $\mathrm{E}_{w} N_{n}(j)$ given the sequence of weights $w(1), w(2), \ldots$

\section{The distribution of $D_{n}$}

\subsection{The basic properties of $D_{n}$}

Let

$$
W_{n}:=\sum_{j=0}^{n} w(j), \quad p_{n}(j):=\frac{w(j)}{W_{n}}, \quad j=0,1, \ldots, n .
$$

Set $f_{0}(t):=1$ and $f_{j}(t):=\mathrm{Ee}^{\mathrm{i} t Y(j)}, j \geq 1$, and let

$$
\begin{gathered}
\varphi_{0}(t):=1, \quad \varphi_{n}(t):=\mathrm{E}_{w} \mathrm{e}^{\mathrm{i} t D_{n}}:=\mathrm{E}\left[\mathrm{e}^{\mathrm{i} t D_{n}} \mid w(1), \ldots, w(n-1)\right], \quad n \geq 1, \\
\Psi_{n}(t):=\mathrm{E} \varphi_{n}(t)=\mathrm{E}^{\mathrm{i} t D_{n}}, \quad n \geq 1
\end{gathered}
$$

(here and in what follows, $\mathrm{E}_{w}$ and $\mathrm{P}_{w}$ respectively denote the conditional expectation and probability given the sequence of weights $\{w(j)\})$.

It is easy to see that

$$
\begin{aligned}
\varphi_{n+1}(t) & =\sum_{j=0}^{n} p_{n}(j) \varphi_{j}(t) f_{n+1}(t) \\
& =\frac{W_{n-1}}{W_{n}} \sum_{j=0}^{n-1} p_{n-1}(j) \varphi_{j}(t) f_{n+1}(t)+p_{n}(n) \varphi_{n}(t) f_{n+1}(t) \\
& =\left(1-p_{n}(n)\right) \frac{f_{n+1}(t)}{f_{n}(t)} \varphi_{n}(t)+p_{n}(n) \varphi_{n}(t) f_{n+1}(t) \\
& =\left[1+\left(f_{n}(t)-1\right) p_{n}(n)\right] \frac{f_{n+1}(t)}{f_{n}(t)} \varphi_{n}(t) \\
& =\cdots \\
& =f_{n+1}(t) \prod_{j=1}^{n}\left[1+\left(f_{j}(t)-1\right) p_{j}(j)\right] .
\end{aligned}
$$

Remark 1. Observe that (2) in fact means that, given the environment, the RV $D_{n+1}$ admits a representation of the form of a sum of independent RVs, as follows:

$$
D_{n+1} \stackrel{\mathrm{D}}{=} I_{1} Y(1)+\cdots+I_{n} Y(n)+Y(n+1) .
$$

Here $\left\{I_{j}\right\}$ is a sequence of random indicators that are independent of each other and also of $\{Y(j)\}$, with $\mathrm{P}\left(I_{j}=1\right)=p_{j}(j), j \geq 1$. In the special case where $Y(j) \equiv w(j) \equiv 1$, this representation is equivalent to the correspondence between the quantity $D_{n}$ and the numbers of records in an i.i.d. sequence that was used in [11] (see also Section 3.6 of [17], for a discussion of a somewhat more general situation where the representation (3) with $Y(j) \equiv 1$ holds). Note, however, that in [11] a probabilistic argument that works only in that special case was used to derive representation (3), which is actually the main tool for studying $D_{n}$, whereas our approach leads directly to (3) and is much more general. 
From the recursive relation (2), we can derive a number of interesting results on the limiting behaviour of $D_{n}$. Note that (2) was first derived in the case where $w(j)=a^{j}, j \geq 0, Y(j) \in$ $\mathbb{R}^{d}$, in [10] (one can easily see that this recursive formula and the statements of Theorems 1 and 2 below remain true in the multivariate case as well). In particular, relation (2) immediately implies the following assertion, describing the limiting behaviour of the conditional distribution of $D_{n}$ (given the weights) when the weight sequence $\{w(j)\}$ 'suggests' new children to attach not too far from the tree's root.

Theorem 1. If

$$
\sum_{j=1}^{\infty} p_{j}(j)<\infty \text { almost surely (a.s.) }
$$

and the distribution of $Y(n)$ has a weak limit as $n \rightarrow \infty$, i.e. for a characteristic function $f(t)$,

$$
\lim _{n \rightarrow \infty} f_{n}(t)=f(t)
$$

then there exists the limit

$$
\lim _{n \rightarrow \infty} \varphi_{n}(t)=\varphi_{\infty}(t):=f(t) \prod_{j=1}^{\infty}\left[1+\left(f_{j}(t)-1\right) p_{j}(j)\right] \quad \text { a.s. }
$$

This result, in turn, implies that $D_{n} \stackrel{\mathrm{D}}{\rightarrow} D_{\infty}$ as $n \rightarrow \infty$, where $D_{\infty}$ is a proper RV with the characteristic function $\mathrm{E} \varphi_{\infty}(t)$.

The next assertion applies in situations where the attachment preferences are spread 'more uniformly' across the tree.

Theorem 2. Let the sequence of $R V s\{Y(j)\}_{j \geq 1}$ be uniformly integrable, and let there exist both a sequence $\left\{h_{n}\right\}$ such that $h_{n} \rightarrow \infty$ as $n \rightarrow \infty$ and an $R V \zeta$ such that the following convergence in distribution occurs as $n \rightarrow \infty$ :

$$
\zeta_{n}:=\frac{1}{h_{n}} \sum_{j=1}^{n} p_{j}(j) \mathrm{E} Y(j) \stackrel{\mathrm{D}}{\rightarrow} \zeta .
$$

Then, for any $t$,

$$
\varphi_{n}\left(\frac{t}{h_{n}}\right) \stackrel{\mathrm{D}}{\rightarrow} \mathrm{e}^{\mathrm{i} t \zeta} .
$$

Remark 2. We can easily see that if, instead of (4), we have $\zeta_{n} \rightarrow \zeta$ a.s. for some RV $\zeta$, then

$$
\lim _{n \rightarrow \infty} \varphi_{n}\left(\frac{t}{h_{n}}\right)=\mathrm{e}^{\mathrm{i} t \zeta} \quad \text { a.s. }
$$

uniformly in $t$ from any compact set.

Proof of Theorem 2. It is not difficult to see that, due to the uniform integrability condition, as $n \rightarrow \infty$,

$$
f_{j}\left(\frac{t}{h_{n}}\right)-1=\mathrm{E} \exp \left\{\frac{\mathrm{i} t Y(j)}{h_{n}}\right\}-1=\frac{1}{h_{n}}(\mathrm{i} t \mathrm{E} Y(j)+o(1))
$$


uniformly in $j \geq 1$ and in $t$ from any compact set. Hence, as $p_{j}(j) \leq 1$, by (2) we have

$$
\begin{aligned}
\varphi_{n}\left(\frac{t}{h_{n}}\right) & =f_{n}\left(\frac{t}{h_{n}}\right) \prod_{j=1}^{n-1}\left[1+\left(f_{j}\left(\frac{t}{h_{n}}\right)-1\right) p_{j}(j)\right] \\
& =\left(1+\varepsilon_{n}(t)\right) \exp \left\{\frac{\mathrm{i} t}{h_{n}} \sum_{j=1}^{n} p_{j}(j) \mathrm{E} Y(j)\right\},
\end{aligned}
$$

where $\varepsilon_{n}(t)=o_{\mathrm{P}}(1)$ as $n \rightarrow \infty$. This clearly implies the assertion of the theorem.

Corollary 1. Under the conditions of Theorem 2,

$$
\lim _{n \rightarrow \infty} \Psi_{n}\left(\frac{t}{h_{n}}\right)=\mathrm{Ee}^{\mathrm{i} t \zeta},
$$

so $D_{n} / h_{n} \stackrel{\mathrm{D}}{\rightarrow} \zeta$ as $n \rightarrow \infty$.

From Theorem 2 we can also easily deduce the following result obtained in [10] (note that, in the special case where $Y(j) \equiv 1$, the result was originally established in [11]).

Corollary 2. If $w(j) \equiv 1, j=0,1,2, \ldots$, the family of $R V s\{Y(j)\}_{j \geq 1}$ is uniformly integrable, and

$$
\frac{1}{n} \sum_{j=1}^{n} \mathrm{E} Y(j) \rightarrow \mu \in \mathbb{R} \quad \text { as } n \rightarrow \infty
$$

then $D_{n} / \ln n \stackrel{\mathrm{P}}{\rightarrow} \mu$.

Proof. In this case clearly $p_{j}(j)=1 /(j+1)$ and, as was shown in Lemma 1(i) of [10], under the above conditions,

$$
\zeta_{n}=\frac{1}{\ln n} \sum_{j=1}^{n} \frac{1}{j+1} \mathrm{E} Y(j) \rightarrow \mu .
$$

The assertion of the corollary thus follows from Theorem 2.

We also obtain the same asymptotics for $D_{n}$ when the weights are random but remain the same 'on average'.

Corollary 3. If $Y(j) \equiv 1, j \geq 1$, and the sequence of random weights $\{w(j)\}$ satisfies the strong law of large numbers, i.e. as $n \rightarrow \infty$,

$$
\frac{1}{n} \sum_{j=1}^{n} w(j) \rightarrow a>0 \quad \text { a.s. }
$$

then $D_{n} / \ln n \stackrel{\mathrm{P}}{\rightarrow} 1$.

Proof. It again suffices to apply (a slightly modified version of) Lemma 1(i) of [10] (this time to the sequences $\left\{y_{n}:=a n W_{n}^{-1}\right\}$ and $\left.\left\{x_{n}:=w(n) / a\right\}\right)$ and use our Theorem 2. 
Remark 3. To obtain a faster-than-logarithmic growth rate for $D_{n}$ (assuming that $Y(j) \equiv 1$ ), the weights $w(j)$ should grow faster than any power function. Indeed, if, say,

$$
w(j)=j^{\alpha} l(j), \quad \alpha \in \mathbb{R},
$$

is a regularly varying function, then clearly

$$
\sum_{j=1}^{\infty} p_{j}(j)<\infty \quad \text { if } \alpha<-1
$$

(meaning that in this case Theorem 1 is applicable), and, by Karamata's theorem, $W_{n} \sim$ $(\alpha+1)^{-1} n^{\alpha+1} l(n)$ if $\alpha>-1$, meaning that $p_{j}(j) \sim 1 /(\alpha+1) j$ and, hence,

$$
\sum_{j=1}^{n} p_{j}(j) \sim \frac{\ln n}{\alpha+1} \quad \text { if } \alpha>-1 .
$$

Thus, in the latter case, $D_{n} / \ln n \stackrel{\mathrm{P}}{\rightarrow} 1 /(\alpha+1)$.

On the other hand, for, say,

$$
w(j)=\alpha j^{\alpha-1} \mathrm{e}^{j^{\alpha}}, \quad \alpha \in(0,1],
$$

we obtain $W_{n} \sim \mathrm{e}^{n^{\alpha}}$ and, hence,

$$
\sum_{j=1}^{n} p_{j}(j) \sim n^{\alpha} .
$$

This example thus shows that, for any $\alpha \in(0,1]$, we can construct a random recursive tree with $D_{n} / n^{\alpha} \stackrel{\mathrm{P}}{\rightarrow} 1$ as $n \rightarrow \infty$.

\subsection{The case of product-form random weights}

In this subsection we will construct and study recursive trees with random vertex weights of the form $w(j)=a_{1} \cdots a_{j}, j \geq 1$ (where the $a_{j}$ are i.i.d. RVs), and unit edge lengths. As will be clearly seen from the proofs below, the main results will also hold in the case of random i.i.d. edge lengths with finite mean (Remark 4). Thus, restricting our attention to the case of unit edge lengths leads to no loss of generality, but makes the exposition more compact and transparent.

Denote by $\mathcal{T}_{n}, n=0,1,2, \ldots$, the set of all rooted recursive trees having $n$ nonrooted vertices and unit edge lengths (that is, $\mathcal{T}_{n}$ consists of the rooted trees whose roots are labelled 0 and whose nonrooted vertices are labelled by numbers $1,2, \ldots, n$ in such a way that, for any nonrooted vertex labelled, say, $j$, the shortest path leading from it to the root traverses only the vertices labelled by numbers less than $j$ ). For a tree $t_{n} \in \mathcal{T}_{n}$, let $t_{n}(j) \in \mathcal{T}_{n+1}$ be the recursive tree which is obtained from $t_{n}$ by adding a vertex labelled $n+1$ as a child of the vertex with the label $j \in\{0,1, \ldots, n\}$.

We can describe the construction of our random recursive tree as follows. First, we run a random walk

$$
S_{0}=0, \quad S_{j}=\theta_{1}+\cdots+\theta_{j}, \quad j \geq 1,
$$

where the $\theta_{j} \stackrel{\mathrm{D}}{=} \theta, j=1,2, \ldots, n$, are i.i.d. RVs. Second, given $S_{j}, j=0,1, \ldots, n$, we construct a (conditional) Markov chain $T_{0}, T_{1}, \ldots, T_{n}$, with $T_{k} \in \mathcal{T}_{k}, k=0,1, \ldots, n$, by assigning the weight $w(j):=\mathrm{e}^{-S_{j}}$ to the vertex labelled $j \geq 0$ (so $w(j)=a_{1} \cdots a_{j}, j \geq 1$, 
in the notation of Section 1, with the $a_{j}:=\mathrm{e}^{-\theta_{j}}$ being i.i.d. RVs), so that now we have, for $r=0,1, \ldots, n$,

$$
\begin{aligned}
W_{r} & =\sum_{q=0}^{r} w(q)=\sum_{q=0}^{r} \mathrm{e}^{-S_{q}}, \\
p_{r}(j) & =\frac{\mathrm{e}^{-S_{j}}}{W_{r}}=\frac{\mathrm{e}^{-S_{j}}}{\sum_{q=0}^{r} \mathrm{e}^{-S_{q}}}, \quad j=0,1, \ldots, r,
\end{aligned}
$$

and then letting, for any $t_{r} \in \mathcal{T}_{r}$,

$$
\begin{array}{r}
\mathrm{P}_{w}\left(T_{r+1}=t_{r}(j) \mid T_{r}=t_{r}\right) \equiv \mathrm{P}\left(T_{r+1}=t_{r}(j) \mid T_{r}=t_{r} ; w(0), w(1), \ldots, w(r)\right):=p_{r}(j), \\
j=0,1, \ldots, r .
\end{array}
$$

The main result of this subsection is the following theorem.

Theorem 3. If

$$
\mathrm{E} \theta=0, \quad \sigma^{2}:=\mathrm{E} \theta^{2}>0, \quad \mathrm{E}|\theta|^{2+\delta}<\infty \text { for some } \delta>0,
$$

then, as $n \rightarrow \infty$,

$$
\zeta_{n}:=\frac{1}{\sqrt{n}} \sum_{j=1}^{n} p_{j}(j) \stackrel{\mathrm{D}}{\rightarrow} \sigma_{m} \max _{0 \leq u \leq 1} B(u), \quad \sigma_{m}:=\sigma \int_{0}^{\infty} \frac{m(\mathrm{~d} y)}{y}<\infty,
$$

where $\{B(u)\}_{u \geq 0}$ is the standard Brownian motion process and the measure $m$ is specified in the proof (see (11)).

Together with Corollary 1 , the above assertion immediately yields the following result.

Corollary 4. Under the conditions of Theorem 3,

$$
\frac{D_{n}}{\sqrt{n}} \stackrel{\mathrm{D}}{\rightarrow} \sigma_{m} \max _{0 \leq u \leq 1} B(u) \quad \text { as } n \rightarrow \infty
$$

In other words, for any $x>0$,

$$
\mathrm{P}\left(D_{n}>\sigma_{m} \sqrt{n} x\right) \rightarrow 2(1-\Phi(x)),
$$

where $\Phi$ is the standard normal distribution function.

Remark 4. It is obvious that the assertion of Corollary 4 remains true in the case of i.i.d. random edge lengths, $Y(j) \geq 0$, with finite, positive mean, the only difference being that $\sigma_{m}$ should be replaced by $\sigma_{m} \mathrm{E} Y(1)$ in its formulation.

Proof of Theorem 3. Let

$$
L_{n}:=\min _{0 \leq k \leq n} S_{k}
$$

Using the proof of Theorem 4.1 of [2], we will show that

$$
\frac{1}{\left|L_{n}\right|} \sum_{j=1}^{n} p_{j}(j) \rightarrow \int_{0}^{\infty} \frac{m(\mathrm{~d} y)}{y}<\infty \text { a.s. }
$$


Since, by the invariance principle,

$$
\frac{\left|L_{n}\right|}{\sqrt{n}} \stackrel{\mathrm{D}}{\rightarrow} \sigma \max _{0 \leq u \leq 1} B(u) \quad \text { as } n \rightarrow \infty,
$$

the assertion of the theorem will then immediately follow.

First, denote by

$$
\gamma_{0}:=0, \quad \gamma_{j+1}:=\min \left\{n>\gamma_{j}: S_{n}<S_{\gamma_{j}}\right\}, \quad j \geq 0,
$$

the strict descending ladder epochs of the random walk $\left\{S_{n}\right\}_{n \geq 0}$. All the RVs introduced are finite a.s., as, in view of (7), $\left\{S_{n}\right\}_{n \geq 0}$ is recurrent.

Let $\left\{X_{n}\right\}_{n \geq 0}$ be a Markov chain defined, for $n=1,2, \ldots$, by

$$
X_{n}:=\mathrm{e}^{\theta_{n}} X_{n-1}+1 .
$$

When $X_{0}^{x}=x>0$ is a fixed value, we will use the notation $\left\{X_{n}^{x}\right\}_{n \geq 0}$. Clearly,

$$
X_{n}^{x}=x \mathrm{e}^{S_{n}}+\sum_{q=1}^{n} \mathrm{e}^{S_{n}-S_{q}}=\mathrm{e}^{S_{n}}\left(x-1+W_{n}\right) .
$$

Set $\gamma:=\gamma_{1}$. Under our assumptions in (7), the expectation E $S_{\gamma}<0$ is finite (see, e.g. Corollary 10, Section 17, of [9]), and the Markov chain $\left\{X_{\gamma_{n}}\right\}_{n \geq 1}$ with transition kernel

$$
M_{\gamma}(x, \cdot):=\mathrm{P}\left(X_{\gamma}^{x} \in \cdot\right), \quad x>0,
$$

has a unique invariant probability measure, $m_{\gamma}$, satisfying (see, e.g. Lemma 5.49 of [13] and page 481 of [2]):

$$
m_{\gamma}(A)=\int_{0}^{\infty} m_{\gamma}(\mathrm{d} x) M_{\gamma}(x, A) .
$$

Moreover, the measure $m$ defined by

$$
m(f):=\frac{1}{\mathrm{E}\left[-S_{\gamma}\right]} \int_{0}^{\infty} \mathrm{E}\left[\sum_{k=0}^{\gamma-1} f\left(X_{k}^{x}\right)\right] m_{\gamma}(\mathrm{d} x)
$$

is an invariant measure for the Markov chain $\left\{X_{n}\right\}_{n \geq 0}$ (see [2]).

Now note that, by virtue of (6) and (10),

$$
\zeta_{n}=\frac{1}{\sqrt{n}} \sum_{j=1}^{n} p_{j}(j)=\frac{1}{\sqrt{n}} \sum_{j=1}^{n} \frac{1}{X_{j}^{1}} .
$$

Let $P_{\delta_{y}}$ be the distribution of the two-dimensional random walk

$$
Z_{n}:=\left(X_{n}, \mathrm{e}^{S_{n}}\right), \quad n \geq 0
$$

(on the group of transformations $x \mapsto a x+b$ of the real line with the composition law $\left.\left(b_{1}, a_{1}\right)\left(b_{2}, a_{2}\right)=\left(b_{1}+a_{1} b_{2}, a_{1} a_{2}\right)\right)$, when $X_{0}=y$. It was shown in the proof of Theorem 4.1 of [2] that if $f \in L^{1}(m)$ then

$$
\lim _{n \rightarrow \infty} \frac{1}{\left|L_{n}\right|} \sum_{j=1}^{n} f\left(X_{j}\right)=\int_{0}^{\infty} f(y) m(\mathrm{~d} y) \quad P_{m_{\gamma}} \text {-a.s. }
$$


where

$$
P_{m_{\gamma}}:=\int_{0}^{\infty} P_{\delta_{y}} m_{\gamma}(\mathrm{d} y)
$$

is the law of the two-dimensional random walk $\left\{Z_{n}\right\}_{n \geq 1}$ when the distribution of $X_{0}$ is $m_{\gamma}$.

For $N=1,2, \ldots$ and $x>0$, let

$$
g_{N}(x):=\frac{1}{x} \mathbf{1}_{\left\{N^{-1} \leq x \leq N\right\}} \leq \frac{1}{x}=: g(x) .
$$

Clearly, for all $x>0$,

$$
g_{N}(x) \nearrow g(x) \text { as } N \rightarrow \infty,
$$

and $g_{N}(x) \in L^{1}(m)$ for each $N=1,2, \ldots$ Therefore, by (12),

$$
\lim _{n \rightarrow \infty} \frac{1}{\left|L_{n}\right|} \sum_{j=1}^{n} g_{N}\left(X_{j}\right)=\int_{0}^{\infty} g_{N}(y) m(\mathrm{~d} y) \quad P_{m_{\gamma}} \text {-a.s. }
$$

On the other hand, for each $N \geq 1$ and any $x>0$,

$$
\begin{aligned}
\frac{1}{\left|L_{n}\right|} \sum_{j=1}^{n} g_{N}\left(X_{j}^{x}\right) & \leq \frac{1}{\left|L_{n}\right|} \sum_{j=1}^{n} g\left(X_{j}^{x}\right)=\frac{1}{\left|L_{n}\right|} \sum_{j=1}^{n} \frac{\mathrm{e}^{-S_{j}}}{x-1+W_{j}} \\
& \leq \frac{1}{\left|L_{n}\right|} \sum_{j=1}^{n} \int_{x-1+W_{j-1}}^{x-1+W_{j}} \frac{\mathrm{d} y}{y}=\frac{1}{\left|L_{n}\right|} \int_{x-1+W_{0}}^{x-1+W_{n}} \frac{\mathrm{d} y}{y} \\
& =\frac{1}{\left|L_{n}\right|}\left[\ln \left(x-1+W_{n}\right)-\ln x\right] \leq \frac{1}{\left|L_{n}\right|}\left[\ln \left(x+n \mathrm{e}^{\left|L_{n}\right|}\right)-\ln x\right] \\
& \leq \frac{1}{\left|L_{n}\right|}\left[\ln n \mathrm{e}^{\left|L_{n}\right|}+\frac{x}{n \mathrm{e}^{\left|L_{n}\right|}}-\ln x\right]=1+\frac{1}{\left|L_{n}\right|}[\ln n+O(1)] \\
& \stackrel{\mathrm{P}}{\rightarrow} 1 \quad \text { as } n \rightarrow \infty,
\end{aligned}
$$

by the invariance principle (see, e.g. [6, Section 2.10]).

Combining (14) with (15) shows that

$$
\sup _{N \geq 1} \int_{0}^{\infty} g_{N}(y) m(\mathrm{~d} y) \leq 1,
$$

which, together with (13), yields

$$
\int_{0}^{\infty} g(y) m(\mathrm{~d} y) \leq 1
$$

Therefore, by (12),

$$
\lim _{n \rightarrow \infty} \frac{1}{\left|L_{n}\right|} \sum_{j=1}^{n} g\left(X_{j}\right)=\int_{0}^{\infty} g(y) m(\mathrm{~d} y)=\int_{0}^{\infty} \frac{\mathrm{d} m(y)}{y} \quad P_{m_{\gamma}} \text {-a.s. }
$$

To see that this convergence holds for all starting points $x>0$, it suffices to observe that $g(z)$ is monotone in $z>0$ and that $X_{j}^{x_{1}}>X_{j}^{x_{2}}, j \geq 1$, for $x_{1}$ and $x_{2}$ with $x_{1}>x_{2}>0$.

This, in view of (8) and (9), completes the proof of Theorem 3. 


\section{The expectations of the outdegrees of vertices}

Let $N_{n}(j)$ be the outdegree of the vertex $v(j), j=0,1, \ldots, n$, in $\mathcal{T}_{n}$, i.e. the number of edges coming out of $v(j)$ in a tree having $n$ nonrooted vertices. Clearly, the RV $N_{n}(j)$ admits the representation (1) and, therefore,

$$
\begin{aligned}
\mathrm{E}_{w} N_{n}(j) & =\mathrm{E}\left[N_{n}(j) \mid w(1), \ldots, w(n-1)\right]=\sum_{k=j+1}^{n} \mathrm{E}_{w} \mathbf{1}_{\left\{v\left(k^{*}\right)=v(j)\right\}} \\
& =\sum_{k=j+1}^{n} p_{k-1}(j)=\mathrm{e}^{-S_{j}} \sum_{k=j}^{n-1} W_{k}^{-1}
\end{aligned}
$$

and

$$
\mathrm{E} N_{n}(j)=\sum_{k=j}^{n-1} \mathrm{Ee}^{-S_{j}} W_{k}^{-1} .
$$

Our aim in this section is to investigate the asymptotic behaviour (as $n \rightarrow \infty$ ) of the expectations $\mathrm{E} N_{n}(j)$ and that of the distributions of the RVs $\mathrm{E}_{w} N_{n}(j)$ in different ranges of the parameter $j$.

\subsection{The asymptotic behaviour of $\mathrm{E} N_{n}(j)$}

In this section we impose weaker restrictions (compared to conditions (7), used in Section 2) on the random walk $S_{n}=\theta_{1}+\cdots+\theta_{n}, n \geq 1$, where $\theta_{j} \stackrel{\text { D }}{=} \theta$ are i.i.d. RVs. Namely, we assume only that Spitzer's condition holds:

There exists a $\rho \in(0,1)$ such that

$$
\frac{1}{n} \sum_{k=1}^{n} \mathrm{P}\left(S_{k}>0\right) \rightarrow \rho \quad \text { as } n \rightarrow \infty .
$$

It is known [12] that this condition is equivalent to Doney's condition,

$$
\mathrm{P}\left(S_{n}>0\right) \rightarrow \rho \quad \text { as } n \rightarrow \infty
$$

(for a further discussion of condition (18), see, e.g. Section 8.9 of [7]).

We will need a number of auxiliary results concerning the random walk $\left\{S_{n}\right\}_{n \geq 0}$. Let

$$
\Gamma_{0}:=0, \quad \Gamma_{j+1}:=\inf \left\{n>\Gamma_{j}: S_{n}>S_{\Gamma_{j}}\right\}, \quad j \geq 0,
$$

be the strict ascending ladder epochs of the random walk $\left\{S_{n}\right\}_{n \geq 0}$. Recall that the $\gamma_{i}, 0=$ $\gamma_{0}<\gamma_{1}<\gamma_{2}<\cdots$, denote the strict descending ladder epochs of the walk. Introduce the two renewal functions

$$
\begin{aligned}
& U(x):=1+\sum_{j=1}^{\infty} \mathrm{P}\left(S_{\Gamma_{j}}<x\right), \quad x>0, \quad U(0)=1, \quad U(x)=0, \quad x<0, \\
& V(x):=\sum_{j=0}^{\infty} \mathrm{P}\left(S_{\gamma_{j}} \geq-x\right), \quad x>0, \quad V(0)=1, \quad V(x)=0, \quad x<0,
\end{aligned}
$$


and set

$$
M_{n}:=\max _{0 \leq k \leq n} S_{k}, \quad \tilde{M}_{n}:=\max _{1 \leq k \leq n} S_{k} .
$$

It is known (see, e.g. Lemma 1 of [14] and Lemma 1 of [19]) that, under condition (18),

$$
\mathrm{E} U(-\theta) \mathbf{1}_{\{-\theta>0\}}=\mathrm{e}^{-\phi}, \quad \mathrm{E} U(x-\theta) \mathbf{1}_{\{x-\theta>0\}}=U(x), \quad x>0,
$$

where

$$
\phi:=\sum_{j=1}^{\infty} \frac{1}{j} \mathrm{P}\left(S_{j}=0\right)<\infty
$$

and

$$
\mathrm{E} V(x+\theta)=V(x), \quad x \geq 0 .
$$

By means of $V(x)$ and $U(x)$ we can specify two sequences of probability measures, $\left\{\mathrm{P}_{n}^{-}\right\}_{n \geq 1}$ and $\left\{\mathrm{P}_{n}^{+}\right\}_{n \geq 1}$, on the $\sigma$-algebras $\left\{\Sigma_{n}:=\sigma\left(S_{1}, \ldots, S_{n}\right)\right\}_{n \geq 1}$, with the corresponding expectations $\left\{\mathrm{E}_{n}^{-}\right\}_{n \geq 1}$ and $\left\{\mathrm{E}_{n}^{+}\right\}_{n \geq 1}$, by setting

$$
\begin{aligned}
& \mathrm{E}_{n}^{-}\left[\psi_{n}\left(S_{1}, \ldots, S_{n}\right)\right]:=\mathrm{e}^{\phi} \mathrm{E}\left[\psi_{n}\left(S_{1}, \ldots, S_{n}\right) U\left(-S_{n}\right) \mathbf{1}_{\left\{\tilde{M}_{n}<0\right\}}\right], \\
& \mathrm{E}_{n}^{+}\left[\psi_{n}\left(S_{1}, \ldots, S_{n}\right)\right]:=\mathrm{E}\left[\psi_{n}\left(S_{1}, \ldots, S_{n}\right) V\left(S_{n}\right) \mathbf{1}_{\left\{L_{n} \geq 0\right\}}\right]
\end{aligned}
$$

for each bounded, measurable function $\psi_{n}\left(x_{1}, \ldots, x_{n}\right)$. It is easy to verify that (19) and (20) imply that each of the sequences $\left\{\mathrm{P}_{n}^{ \pm}\right\}_{n \geq 1}$ is consistent, and that by Kolmogorov's extension theorem there therefore exist measures $\mathrm{P}^{-}$and $\mathrm{P}^{+}$on the $\sigma$-algebra $\sigma\left(S_{1}, S_{2}, \ldots\right)$ such that their restrictions, $\left.\mathrm{P}^{ \pm}\right|_{\Sigma_{n}}$, to $\Sigma_{n}$ coincide with $\mathrm{P}_{n}^{ \pm}, n=1,2, \ldots$

It is known (see Lemma 2.7 of [1]) that, under condition (18),

$$
\eta_{1}:=\sum_{k=1}^{\infty} \mathrm{e}^{S_{k}}<\infty \quad \mathrm{P}^{-} \text {-a.s., } \quad \eta_{2}:=\sum_{k=0}^{\infty} \mathrm{e}^{-S_{k}}<\infty \quad \mathrm{P}^{+} \text {-a.s. }
$$

Finally, it is not difficult to deduce from Lemma 3 of [19] that, if we let

$$
H_{n}^{-}(x):=\mathrm{P}\left(\sum_{k=1}^{n} \mathrm{e}^{S_{k}} \leq x \mid \tilde{M}_{n}<0\right), \quad H_{n}^{+}(x):=\mathrm{P}\left(\sum_{k=0}^{n} \mathrm{e}^{-S_{k}} \leq x \mid L_{n} \geq 0\right)
$$

and

$$
H^{-}(x):=\mathrm{P}^{-}\left(\eta_{1}<x\right), \quad H^{+}(x):=\mathrm{P}^{+}\left(\eta_{2}<x\right),
$$

then, under condition (18),

$$
H_{n}^{ \pm}(x) \Rightarrow H^{ \pm}(x) \quad \text { as } n \rightarrow \infty
$$

where the symbol ' $\Rightarrow$ ' denotes convergence at all continuity points of the limiting function.

In what follows we will often use the following result (see, e.g. Lemma 2.1 of [1], Theorem 8.9.12 of [7], and Lemma 2 of [19]). Let

$$
\lambda_{n}(x):=\mathrm{P}\left(L_{n} \geq-x\right), \quad \tilde{\mu}_{n}(x):=\mathrm{P}\left(\tilde{M}_{n}<x\right), \quad x \geq 0 .
$$


Lemma 1. Under Spitzer's condition, (18), there exist functions $l_{1}(n)$ and $l_{2}(n)$, related by $l_{1}(n) l_{2}(n) \sim \pi^{-1} \sin \pi \rho, n \rightarrow \infty$, that are slowly varying at infinity and such that

$$
\mathrm{P}\left(L_{n} \geq 0\right) \sim n^{\rho-1} l_{1}(n) \text { and } \mathrm{P}\left(\tilde{M}_{n}<0\right) \sim n^{-\rho} l_{2}(n), \quad \text { as } n \rightarrow \infty .
$$

Moreover, there exist absolute constants $C_{1}>0$ and $C_{2}>0$ such that, for all $n \geq 1$ and $x \geq 0$,

$$
\lambda_{n}(x) \leq C_{1} V(x) \mathrm{P}\left(L_{n} \geq 0\right), \quad \tilde{\mu}_{n}(x) \leq C_{2} U(x) \mathrm{P}\left(\tilde{M}_{n}<0\right) .
$$

In (25) and in the rest of the paper, by $a_{n} \sim b_{n}$ we mean that $a_{n} / b_{n} \rightarrow 1$ as $n \rightarrow \infty$.

Let $\left\{S_{n}^{-}\right\}_{n \geq 0}$ and $\left\{S_{n}^{+}\right\}_{n \geq 0}$ be two independent copies of $\left\{S_{n}\right\}_{n \geq 0}$, and let

$$
L_{n}^{+}:=\min _{0 \leq r \leq n} S_{r}^{+}, \quad \tilde{M}_{n}^{-}:=\max _{1 \leq l \leq n} S_{l}^{-} .
$$

Introduce the probability distributions

$$
\mathrm{P}_{-,+}:=\mathrm{P}^{-} \times \mathrm{P}^{+}, \quad \mathrm{P}_{,++}:=\mathrm{P} \times \mathrm{P}^{+}, \quad \mathrm{P}_{-, .}:=\mathrm{P}^{-} \times \mathrm{P}
$$

on the sample space $\mathbb{R}^{\infty} \times \mathbb{R}^{\infty}$ of the pair $\left(\left\{S_{n}^{-}\right\}_{n \geq 0},\left\{S_{n}^{+}\right\}_{n \geq 0}\right)$, where $\mathrm{P}$ is the distribution of the original sequence $\left\{S_{n}\right\}_{n \geq 0}$ and the measures $\mathrm{P}^{ \pm}$are specified by (21) and (22), and let $\mathrm{E}_{-,+}$,

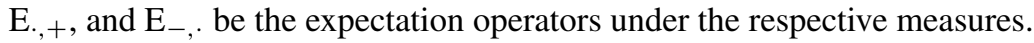

We will call an array of RVs $\left\{G_{l, r}: l, r \in \mathbb{N}\right\}$ adapted if, for any pair of indices $l, r \in \mathbb{N}$, the $\mathrm{RV} G_{l, r}$ is measurable with respect to the $\sigma$-algebra $\sigma\left(S_{1}^{-}, \ldots, S_{l}^{-}\right) \otimes \sigma\left(S_{1}^{+}, \ldots, S_{r}^{+}\right)$. The following result is contained in Lemma 3 of [19].

Lemma 2. Let Spitzer's condition, (18), hold, and let $\left\{G_{l, r}: l, r \in \mathbb{N}\right\}$ be an adapted array of uniformly bounded RVs. If the limit

$$
\lim _{l, r \rightarrow \infty} G_{l, r}=: G \quad \mathrm{P}_{-,+}-\text {a.s. }
$$

exists then

$$
\lim _{l, r \rightarrow \infty} \mathrm{E}\left[G_{l, r} \mid \tilde{M}_{l}^{-}<0, L_{r}^{+} \geq 0\right]=\mathrm{E}_{-,+} G .
$$

The next statement is a slight modification of Lemma 2.5 of [1] and can be proved using the same arguments used there.

Lemma 3. Let Spitzer's condition, (18), hold, and let $\left\{G_{l, r}: l, r \in \mathbb{N}\right\}$ be an adapted array of uniformly bounded RVs. If the limit

$$
\lim _{r \rightarrow \infty} G_{l, r} \mathbf{1}_{\left\{\tilde{M}_{l}^{-}<0\right\}}=: G_{l}^{+} \mathbf{1}_{\left\{\tilde{M}_{l}^{-}<0\right\}} \quad \mathrm{P} \cdot,++ \text {-a.s. }
$$

exists, then

$$
\lim _{r \rightarrow \infty} \mathrm{E}\left[G_{l, r} \mathbf{1}_{\left\{\tilde{M}_{l}^{-}<0\right\}} \mid L_{r}^{+} \geq 0\right]=\mathrm{E}_{\cdot,+} G_{l}^{+} \mathbf{1}_{\left\{\tilde{M}_{l}^{-}<0\right\}}
$$

and if the limit

$$
\lim _{l \rightarrow \infty} G_{l, r} \mathbf{1}_{\left\{L_{r}^{+} \geq 0\right\}}=: G_{r}^{-} \mathbf{1}_{\left\{L_{r}^{+} \geq 0\right\}} \quad \mathrm{P}_{-, .} \text {-a.s. }
$$

exists, then

$$
\lim _{l \rightarrow \infty} \mathrm{E}\left[G_{l, r} \mathbf{1}_{\left\{L_{r}^{+} \geq 0\right\}} \mid \tilde{M}_{l}^{-}<0\right]=\mathrm{E}_{-, .} G_{r}^{-} \mathbf{1}_{\left\{L_{r}^{+} \geq 0\right\}} .
$$


The following result was proved in Lemma 2.2 of [1]. Denote by

$$
\tau(n):=\min \left\{k \geq 0: S_{k} \leq S_{l}, l \in[0, n]\right\}
$$

the left-most point at which the random walk $\left\{S_{n}\right\}$ attains its minimum value on the time interval $[0, n]$.

Lemma 4. Let Spitzer's condition, (18), hold, and let $u(x) \geq 0, x \geq 0$, be a nonincreasing function such that $\int_{0}^{\infty} u(x) \mathrm{d} x<\infty$. Then, for every $\varepsilon>0$, there exists an integer $J$ such that, for all $n \geq J$,

$$
\sum_{p=J}^{n} \mathrm{E}\left[u\left(-S_{p}\right) ; \tau(p)=p\right] \mathrm{P}\left(L_{n-p} \geq 0\right) \leq \varepsilon \mathrm{P}\left(L_{n} \geq 0\right) .
$$

Introduce the RVs

$$
\begin{aligned}
G_{r}^{+}(j) & :=\frac{\mathrm{e}^{-S_{j-r}^{+}} \mathbf{1}_{\{j \geq r\}}+\mathrm{e}^{S_{r-j}^{-}} \mathbf{1}_{\{j<r\}}}{\sum_{p=1}^{r} \mathrm{e}^{S_{p}^{-}}+\eta_{2}^{+}}, \\
G_{r}^{-}(j) & :=\frac{\mathrm{e}^{S_{j-r}^{-}} \mathbf{1}_{\{j>r\}}+\mathrm{e}^{-S_{r-j}^{+}} \mathbf{1}_{\{j \leq r\}}}{\eta_{1}^{-}+\sum_{p=0}^{r} \mathrm{e}^{-S_{p}^{+}}},
\end{aligned}
$$

where $\eta_{1}^{-}$and $\eta_{2}^{+}$are defined as in (23), but for the random walks $\left\{S_{n}^{-}\right\}_{n \geq 0}$ and $\left\{S_{n}^{+}\right\}_{n \geq 0}$, respectively. Note that $0<G_{r}^{ \pm} \leq 1$ and, in view of (23), that $G_{r}^{+}(j)$ and $G_{r}^{-}(j)$ are a.s. positive under the measures $\mathrm{P}_{.,+}$and $\mathrm{P}_{-, .}$, respectively. Set

$$
\tilde{L}_{n}^{+}:=\min _{1 \leq p \leq n} S_{p}^{+}
$$

and let

$$
c_{j}:=\sum_{l=0}^{\infty} \mathrm{E}_{,,+} G_{l}^{+}(j) \mathbf{1}_{\left\{\tilde{M}_{l}^{-}<0\right\}}, \quad d_{j}:=\sum_{q=1}^{j} \sum_{r=0}^{\infty} \mathrm{E}_{-, .} G_{r}^{-}(q) \mathbf{1}_{\left\{\tilde{L}_{r}^{+}>0\right\}} .
$$

We can easily verify that $c_{j}$ and $d_{j}$ are finite for any $j=0,1, \ldots$ Thus,

$$
\begin{aligned}
c_{j} \leq j+1+\sum_{l=j+1}^{\infty} \mathrm{E}_{,}+\mathrm{e}^{S_{l-j}^{-}} \mathbf{1}_{\left\{\tilde{M}_{l}^{-}<0\right\}} & =j+1+\sum_{p=1}^{\infty} \mathrm{Ee}^{S_{p}} \mathbf{1}_{\left\{\tilde{M}_{p}<0\right\}} \\
& =j+1+\sum_{p=1}^{\infty} \mathrm{Ee}^{S_{p}} \mathbf{1}_{\left\{S_{1}<0, \ldots, S_{p}<0\right\}} \\
& <\infty
\end{aligned}
$$

(see D2, Section 17, of [18]).

Now we are ready to formulate and prove the following statement.

Theorem 4. Let Spitzer's condition, (18), hold. Then, for any fixed $j \geq 0$,

$$
\begin{aligned}
& \lim _{n \rightarrow \infty} \frac{\mathrm{E} N_{n}(j)}{n \mathrm{P}\left(L_{n} \geq 0\right)}=\frac{c_{j}}{\rho}, \\
& \lim _{n \rightarrow \infty} \frac{\mathrm{E} N_{n}(n-j)}{\mathrm{P}\left(\tilde{M}_{n}<0\right)}=d_{j} .
\end{aligned}
$$


Remark 5. In view of (25), the relations (29) and (30) can be rewritten as follows:

$$
\mathrm{E} N_{n}(j) \sim c_{j} \rho^{-1} n^{\rho} l_{1}(n) \quad \text { and } \quad \mathrm{E} N_{n}(n-j) \sim d_{j} n^{-\rho} l_{2}(n), \quad \text { as } n \rightarrow \infty .
$$

Proof of Theorem 4. To prove the theorem, we have to evaluate the sum, (17), of expectations of the form

$$
\mathrm{Ee}^{-S_{j}} W_{k}^{-1}=\sum_{l=0}^{k} \mathrm{Ee}^{-S_{j}} W_{k}^{-1} \mathbf{1}_{\{\tau(k)=l\}} .
$$

The key idea both in this proof and in that of Theorem 5 is quite similar to that of the Laplace method: the main contribution to the expectation (31) comes from the event where $j$ is close to $\tau(k)$ (for other values of $j \leq k$, the quantity $\mathrm{e}^{-S_{j}}$ will typically be quite small compared with $W_{k}$ ).

First, we will show that, for each fixed $\varepsilon>0$, there exists a $J \equiv J(\varepsilon)$ such that, for all $j \geq 0$ and all $k \geq J+j$,

$$
\mathrm{Ee}^{-S_{j}} W_{k}^{-1} \mathbf{1}_{\{\tau(k) \geq J+j\}} \leq \varepsilon \mathrm{P}\left(L_{k-j} \geq 0\right)
$$

Indeed, as $W_{k} \geq \mathrm{e}^{-S_{\tau(k)}}$, we have

$$
\begin{aligned}
\mathrm{Ee}^{-S_{j}} W_{k}^{-1} \mathbf{1}_{\{\tau(k) \geq J+j\}} & \leq \mathrm{Ee}^{S_{\tau(k)}-S_{j}} \mathbf{1}_{\{\tau(k) \geq J+j\}} \\
& =\sum_{p=J}^{k-j} \mathrm{Ee}^{S_{p+j}-S_{j}} \mathbf{1}_{\{\tau(k)=p+j\}} \\
& \leq \sum_{p=J}^{k-j} \mathrm{Ee}^{S_{p}} \mathbf{1}_{\{\tau(k-j)=p\}} \\
& \left.=\sum_{p=J}^{k-j} \mathrm{E}^{\mathrm{e}^{S_{p}}} \mathbf{1}_{\{\tau(p)=p\}}\right] \mathrm{P}\left(L_{k-j-p} \geq 0\right),
\end{aligned}
$$

and to obtain the result required it remains to apply Lemma 4 with $u(x)=\mathrm{e}^{-x}$.

The next step is to demonstrate that, for any fixed $j \geq 0$ and $l \geq 1$,

$$
\lim _{k \rightarrow \infty} \frac{\mathrm{Ee}^{-S_{j}} W_{k}^{-1} \mathbf{1}_{\{\tau(k)=l\}}}{\mathrm{P}\left(L_{k} \geq 0\right)}=\mathrm{E}_{.,+} G_{l}^{+}(j) \mathbf{1}_{\left\{\tilde{M}_{l}^{-}<0\right\}} .
$$

However, this is an easy consequence of Lemma 3. Indeed, assume first that $j \geq l$. Then, for the RVs $G_{l, r}(j)$ defined for $r \geq j-l$ by

$$
G_{l, k-l}(j):=\frac{\mathrm{e}^{-S_{j-l}^{+}}}{\sum_{p=1}^{l} \mathrm{e}^{S_{p}^{-}}+\sum_{q=0}^{k-l} \mathrm{e}^{-S_{q}^{+}}} \leq 1, \quad k \geq j
$$

(for $r<j-l$ we can set $G_{l, r}(j) \equiv 1$ ), we have

$$
\begin{aligned}
\mathrm{Ee}^{-S_{j}} W_{k}^{-1} \mathbf{1}_{\{\tau(k)=l\}} & =\mathrm{E} \frac{\mathrm{e}^{S_{\tau(k)}-S_{j}}}{\sum_{p=0}^{k} \mathrm{e}^{S_{\tau(k)}-S_{p}}} \mathbf{1}_{\{\tau(k)=l\}} \\
& =\mathrm{E} G_{l, k-l}(j) \mathbf{1}_{\left\{\tilde{M}_{l}^{-}<0, L_{k-l}^{+} \geq 0\right\}} \\
& =\mathrm{E}\left[G_{l, k-l}(j) \mathbf{1}_{\left\{\tilde{M}_{l}^{-}<0\right\}} \mid L_{k-l}^{+} \geq 0\right] \mathrm{P}\left(L_{k-l} \geq 0\right)
\end{aligned}
$$


(here the second relation follows from the duality principle: we use the 'time-reversed random walk' on $[0, l])$.

It is evident that, as $k \rightarrow \infty$,

$$
G_{l, k-l}(j) \mathbf{1}_{\left\{\tilde{M}_{l}^{-}<0\right\}} \rightarrow G_{l}^{+}(j) \mathbf{1}_{\left\{\tilde{M}_{l}^{-}<0\right\}} \quad \mathrm{P}_{\cdot,+} \text {-a.s. }
$$

and, therefore, by Lemma 3, that

$$
\lim _{k \rightarrow \infty} \mathrm{E}\left[G_{l, k-l}(j) \mathbf{1}_{\left\{\tilde{M}_{l}^{-}<0\right\}} \mid L_{k-l}^{+} \geq 0\right]=\mathrm{E}_{.,+} G_{l}^{+}(j) \mathbf{1}_{\left\{\tilde{M}_{l}^{-}<0\right\}} .
$$

On the other hand, in view of (25), for each fixed $l$ we have

$$
\lim _{k \rightarrow \infty} \frac{\mathrm{P}\left(L_{k-l} \geq 0\right)}{\mathrm{P}\left(L_{k} \geq 0\right)}=1 .
$$

Combining this with (34) gives (33). The case where $j<l$ can be treated in a similar way.

Now everything is ready to complete the proof of the first part of the theorem. It follows from (31), (32), and (33) that, for each fixed $j \geq 0$,

$$
\mathrm{Ee}^{-S_{j}} W_{k}^{-1} \sim c_{j} \mathrm{P}\left(L_{k} \geq 0\right) \quad \text { as } k \rightarrow \infty .
$$

Therefore, for a fixed $\varepsilon>0$ there exists a $K(\varepsilon)<\infty$ such that, for all $K \geq K(\varepsilon)$ and $n>K$,

$$
\begin{aligned}
(1-\varepsilon) c_{j} \sum_{k=K+1}^{n-1} \mathrm{P}\left(L_{k} \geq 0\right) \leq \mathrm{E} N_{n}(j) & =\sum_{k=j+1}^{K} \mathrm{Ee}^{-S_{j}} W_{k}^{-1}+\sum_{k=K+1}^{n-1} \mathrm{Ee}^{-S_{j}} W_{k}^{-1} \\
& \leq(K-j)+(1+\varepsilon) c_{j} \sum_{k=K+1}^{n-1} \mathrm{P}\left(L_{k} \geq 0\right) .
\end{aligned}
$$

By (25) and Karamata's theorem (see, e.g. Section 1.6 of [7]),

$$
\sum_{k=K+1}^{n-1} \mathrm{P}\left(L_{k} \geq 0\right) \sim \frac{n}{\rho} \mathrm{P}\left(L_{n} \geq 0\right) \quad \text { as } n \rightarrow \infty .
$$

This together with (37) completes the proof of (29).

Now we will prove (30). Let $\left\{S_{n}^{*}\right\}_{n \geq 0} \stackrel{\text { D }}{=}\left\{-S_{n}\right\}_{n \geq 0}$ be the 'reflected' random walk. By the duality principle, for each fixed $q \leq j$,

$$
\begin{aligned}
\mathrm{Ee}^{-S_{n-j} W_{n-q}^{-1}} & =\mathrm{E} \frac{\mathrm{e}^{-S_{n-j}}}{\sum_{p=0}^{n-q} \mathrm{e}^{-S_{n-q-p}}}=\mathrm{E} \frac{\mathrm{e}^{S_{n-q}-S_{n-j}}}{\sum_{p=0}^{n-q} \mathrm{e}^{S_{n-q}-S_{n-q-p}}} \\
& =\mathrm{E} \frac{\mathrm{e}^{-S_{j-q}^{*}}}{\sum_{p=0}^{n-q} \mathrm{e}^{-S_{p}^{*}}}=\mathrm{Ee}^{-S_{j-q}^{*}\left(W_{n-q}^{*}\right)^{-1}}
\end{aligned}
$$

(with an obvious definition for $W_{n-q}^{*}$ ).

Next we set

$$
L_{n}^{*}:=\min _{0 \leq k \leq n} S_{k}^{*}, \quad \tilde{M}_{n}^{*}:=\max _{1 \leq k \leq n} S_{k}^{*}
$$


and observe that, as $n \rightarrow \infty$,

$$
\mathrm{P}\left(L_{n}^{*} \geq 0\right)=\mathrm{P}\left(M_{n} \leq 0\right) \sim \mathrm{e}^{\phi} \mathrm{P}\left(\tilde{M}_{n}<0\right) .
$$

Indeed, by setting

$$
\chi:=\inf \left\{k \geq 1: S_{k} \geq 0\right\}, \quad \tilde{\chi}:=\inf \left\{k \geq 1: S_{k}>0\right\},
$$

we find from the factorization identities that, for $|z|<1$,

$$
1-\mathrm{E} z^{\tilde{\chi}}=\exp \left\{\sum_{n=0}^{\infty} \frac{z^{n}}{n} \mathrm{P}\left(S_{n}>0\right)\right\}, \quad 1-\mathrm{E} z^{\chi}=\exp \left\{\sum_{n=0}^{\infty} \frac{z^{n}}{n} \mathrm{P}\left(S_{n} \geq 0\right)\right\}
$$

(see, e.g. Corollary 4, Section 16, of [9]). Dividing both sides of these identities by $1-z=$ $\mathrm{e}^{\ln (1-z)}$ yields

$$
\begin{aligned}
\sum_{n=0}^{\infty} z^{n} \mathrm{P}\left(M_{n} \leq 0\right) & =\sum_{n=0}^{\infty} z^{n} \mathrm{P}(\tilde{\chi}>n)=\frac{1-\mathrm{E} z^{\tilde{\chi}}}{1-z} \\
& =\exp \left\{-\sum_{n=1}^{\infty} \frac{z^{n}}{n} \mathrm{P}\left(S_{n}>0\right)+\sum_{n=1}^{\infty} \frac{z^{n}}{n}\right\} \\
& =\exp \left\{\sum_{n=1}^{\infty} \frac{z^{n}}{n} \mathrm{P}\left(S_{n} \leq 0\right)\right\}
\end{aligned}
$$

and, similarly,

$$
\sum_{n=0}^{\infty} z^{n} \mathrm{P}\left(\tilde{M}_{n}<0\right)=\sum_{n=0}^{\infty} z^{n} \mathrm{P}(\chi>n)=\exp \left\{\sum_{n=1}^{\infty} \frac{z^{n}}{n} \mathrm{P}\left(S_{n}<0\right)\right\} .
$$

Therefore,

$$
\sum_{n=0}^{\infty} z^{n} \mathrm{P}\left(M_{n} \leq 0\right)=\mathrm{e}^{\phi(z)} \sum_{n=0}^{\infty} z^{n} \mathrm{P}\left(\tilde{M}_{n}<0\right), \quad \phi(z):=\sum_{n=1}^{\infty} \frac{z^{n}}{n} \mathrm{P}\left(S_{n}=0\right) .
$$

To prove (39), it remains to use (25) and Karamata's Tauberian theorem (see, e.g. Corollary 1.7.3 of [7]), noting that $\phi(z) \rightarrow \phi$ as $z \nearrow 1$.

Now, from (36) and (39) we find that, as $n \rightarrow \infty$,

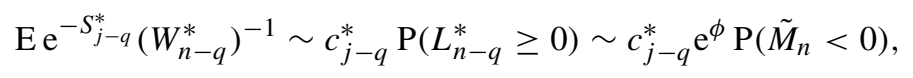

where, with a natural definition of $E_{\cdot,+}^{*}$ and with $\tilde{L}_{r}^{+}$as defined in (27), from definitions (21) and (22), we have

$$
\mathrm{e}^{\phi} c_{j-q}^{*}=\mathrm{e}^{\phi} \sum_{l=0}^{\infty} \mathrm{E}_{\cdot,+}^{*} G_{l}^{*+}(j-q) \mathbf{1}_{\left\{\tilde{M}_{l}^{*-}<0\right\}}=\sum_{r=0}^{\infty} \mathrm{E}_{-, .} G_{r}^{-}(j-q) \mathbf{1}_{\left\{\tilde{L}_{r}^{+}>0\right\}} .
$$


Therefore, from (17) and (38), as $n \rightarrow \infty$ we have

$$
\begin{aligned}
\mathrm{E} N_{n}(n-j) & =\sum_{k=n-j}^{n-1} \mathrm{Ee}^{-S_{n-j}} W_{k}^{-1}=\sum_{q=1}^{j} \mathrm{Ee}^{-S_{j-q}^{*}\left(W_{n-q}^{*}\right)^{-1}} \\
& \sim \mathrm{P}\left(L_{n}^{*} \geq 0\right) \sum_{q=1}^{j} c_{j-q}^{*} \sim \mathrm{P}\left(L_{n}^{*} \geq 0\right) \mathrm{e}^{-\phi} \sum_{q=1}^{j} \sum_{r=0}^{\infty} \mathrm{E}_{-,}, G_{r}^{-}(j-q) \mathbf{1}_{\left\{\tilde{L}_{r}^{+}>0\right\}} \\
& \sim d_{j} \mathrm{P}\left(\tilde{M}_{n}<0\right),
\end{aligned}
$$

as desired. Theorem 4 is thus proved.

The next theorem describes the asymptotic behaviour of the expectation $\mathrm{E} N_{n}(j)$ as

$$
\min \{j, n-j\} \rightarrow \infty
$$

Theorem 5. Let Spitzer's condition, (18), be satisfied. Then

$$
\lim _{j, n-j \rightarrow \infty} \frac{\mathrm{E} N_{n}(j)}{(n-j) \mathrm{P}\left(\tilde{M}_{j}<0\right) \mathrm{P}\left(L_{n-j} \geq 0\right)}=\frac{1}{\rho} .
$$

Remark 6. In view of (25), the assertion of the theorem can be rewritten as follows:

$$
\mathrm{E} N_{n}(j) \sim \rho^{-1} j^{-\rho} l_{2}(j)(n-j)^{\rho} l_{1}(n-j) \quad \text { as } j, n-j \rightarrow \infty .
$$

It follows that, for any fixed $\varepsilon \in\left(0, \frac{1}{2}\right)$, we have, for $t \in[\varepsilon, 1-\varepsilon]$,

$$
\mathrm{E} N_{n}(\lfloor n t\rfloor) \sim \frac{\sin \pi \rho}{\pi \rho}\left(\frac{1-t}{t}\right)^{\rho} \text { as } n \rightarrow \infty,
$$

where $\lfloor x\rfloor$ denotes the integer part of $x$. It is interesting to compare this with the corresponding (obvious) asymptotics for the case $w(j) \equiv 1$, where $\mathrm{E} N_{n}(\lfloor n t\rfloor) \sim-\ln t$ (of course, the functions of $t$ on the right-hand sides of the respective relations are densities on $(0,1))$.

In the case where $\mathrm{E} \theta=0$ and $\mathrm{E} \theta^{2}<\infty$, we do not even need to bound the value $j / n$ away from 0 and 1: in that case, from the asymptotic behaviour of the denominators in (40) (see, e.g. page 94 of [9]), we have

$$
\mathrm{E} N_{n}(j) \sim \frac{2}{\pi}\left(\frac{n-j}{j}\right)^{1 / 2} \text { as } j, n-j \rightarrow \infty .
$$

Note also that assertions (29) and (30) (Theorem 4) can be viewed as the 'boundary cases' of (40): there is a 'smooth transition' between these asymptotics. To make the meaning of the last statement more precise, we also have to show that $c_{j} \sim \mathrm{P}\left(\tilde{M}_{j}<0\right)$ as $j \rightarrow \infty$, which is a separate, nontrivial problem that is somewhat beyond the scope of the present paper. This claim, however, is more than plausible, as can be seen from the following simple (but not quite rigorous) argument. When $j$ is large, the main contribution to the sum in $c_{j}$ in (28) comes from terms with $l$ close to $j$. Indeed, for $l-j \gg 1$, the $l$ th term in that sum is

$$
\mathrm{E} .,\left[\frac{\mathrm{e}^{S_{l-j}^{-}}}{\sum_{p=1}^{l} \mathrm{e}^{S_{p}^{-}}+\eta_{2}^{+}} \mid \tilde{M}_{l}^{-}<0\right] \mathrm{P}\left(\tilde{M}_{l}^{-}<0\right) \sim \mathrm{E}_{-,+}\left[\frac{\mathrm{e}^{S_{l-j}^{-}}}{\eta_{1}^{-}+\eta_{2}^{+}}\right] \mathrm{P}\left(\tilde{M}_{l}^{-}<0\right),
$$


where the equivalence is due to the following interpretation: using $\mathrm{P}^{-}$corresponds to conditioning our random walk to stay negative; see, e.g. [4]. This interpretation also implies that the contribution of all such terms to the sum $c_{j}$ will be relatively small, as, for large $l-j$, the sums $S_{l-j}^{-}$typically assume fairly large negative values, given that the walk stays negative. A similar assertion holds when $j-l \gg 1$. On the other hand, for the terms with indices $l$ that are 'close' to $j$ (assume, say, that $|l-j| \leq K$ for an arbitrarily large, fixed $K$ ), we could use the fact that $\mathrm{P}\left(\tilde{M}_{l}^{-}<0\right) \sim \mathrm{P}\left(\tilde{M}_{j}^{-}<0\right)$ as $j \rightarrow \infty$ and $l / j \rightarrow 1$ (due to regular variation), and then observe that the sum of the conditional expectations that appear on the left-hand side of (41) will be close to 1 . This is because there we are basically conditioning on the random walk $\left\{S_{k}^{-}\right\}$staying negative, and, under $\mathrm{E}_{-,+}$, for large $K$ and $j \gg K$ we have

$$
\sum_{|l-j| \leq K} \frac{\mathrm{e}^{-S_{j-l}^{+}} \mathbf{1}_{\{j \geq l\}}+\mathrm{e}^{S_{l-j}^{-}} \mathbf{1}_{\{j<l\}}}{\sum_{p=1}^{l} \mathrm{e}^{S_{p}^{-}}+\eta_{2}^{+}} \approx \frac{1}{\eta_{1}^{-}+\eta_{2}^{+}}\left[\sum_{k=0}^{K} \mathrm{e}^{-S_{k}^{+}}+\sum_{k=1}^{K} \mathrm{e}^{S_{k}^{-}}\right] \approx 1 .
$$

We split the proof of the theorem into several steps. As said above, the main contribution to the expectation $\mathrm{E} \mathrm{e}^{-S_{j}} W_{k}^{-1}$ from the sum (17) comes from the event where $j$ is close to $\tau(k)$. So first we will show that the contribution from the complementary event is negligibly small.

Lemma 5. Under Spitzer's condition, (18), for any $\varepsilon>0$ there exists a $J \equiv J(\varepsilon)<\infty$ such that, for all $j \geq J$ and $k-j \geq J$,

$$
\mathrm{E}\left[\mathrm{e}^{S_{\tau(k)}-S_{j}} ;|\tau(k)-j| \geq J\right] \leq \varepsilon \mathrm{P}\left(\tilde{M}_{j}<0\right) \mathrm{P}\left(L_{k-j} \geq 0\right) .
$$

Proof. Fix a $J>0$ and choose a $j \geq J$ and a $k \geq j+J$. We have

$$
\mathrm{E}\left[\mathrm{e}^{S_{\tau(k)}-S_{j}} ;|\tau(k)-j| \geq J\right]=R_{1}+R_{2},
$$

where

$$
R_{1}:=\sum_{t=0}^{j-J} \mathrm{E}\left[\mathrm{e}^{S_{\tau(k)}-S_{j}} ; \tau(k)=t\right], \quad R_{2}:=\sum_{t=j+J}^{k} \mathrm{E}\left[\mathrm{e}^{S_{\tau(k)}-S_{j}} ; \tau(k)=t\right] .
$$

First consider $R_{2}$. For $t \geq j$, we obtain

$$
\begin{aligned}
\mathrm{E}\left[\mathrm{e}^{S_{\tau(k)}-S_{j}} ; \tau(k)=t\right] & =\mathrm{E}\left[\mathrm{e}^{S_{t}-S_{j}} ; \min _{0 \leq p \leq t-1} S_{p}>S_{t}, \min _{t \leq p \leq k} S_{p} \geq S_{t}\right] \\
& =\mathrm{E}\left[\mathrm{e}^{S_{t}-S_{j}} ; \min _{0 \leq p \leq t-1} S_{p}>S_{t}\right] \mathrm{P}\left(L_{k-t} \geq 0\right) \\
& =\mathrm{E}\left[\mathrm{e}^{S_{t-j}} ; \max _{1 \leq p \leq t} S_{p}<0\right] \mathrm{P}\left(L_{k-t} \geq 0\right),
\end{aligned}
$$

by the duality principle. Defining for each $l \geq 0$ the shifted random walk

$$
\left\{S_{p}^{(l)}:=S_{l+p}-S_{l}\right\}_{p \geq 0},
$$

from (26) we obtain

$$
\begin{aligned}
\mathrm{E}\left[\mathrm{e}^{S_{t-j}} ; \max _{1 \leq p \leq t} S_{p}<0\right] & =\mathrm{E}\left[\mathrm{e}^{S_{t-j}} \mathrm{P}\left(\max _{1 \leq p \leq j} S_{p}^{(t-j)}<-S_{t-j} \mid S_{t-j}\right) ; \max _{1 \leq p \leq t-j} S_{p}<0\right] \\
& =\mathrm{E}\left[\mathrm{e}^{S_{t-j}} \tilde{\mu}_{j}\left(-S_{t-j}\right) ; \tilde{M}_{t-j}<0\right] \\
& \leq C_{2} \mathrm{P}\left(\tilde{M}_{j}<0\right) \mathrm{E}\left[\mathrm{e}^{S_{t-j}} U\left(-S_{t-j}\right) ; \tilde{M}_{t-j}<0\right] .
\end{aligned}
$$


Hence,

$$
\begin{aligned}
R_{2} & \leq C_{2} \mathrm{P}\left(\tilde{M}_{j}<0\right) \sum_{t=j+J}^{k} \mathrm{E}\left[\mathrm{e}^{S_{t-j}} U\left(-S_{t-j}\right) ; \tilde{M}_{t-j}<0\right] \mathrm{P}\left(L_{k-t} \geq 0\right) \\
& =C_{2} \mathrm{P}\left(\tilde{M}_{j}<0\right) \sum_{p=J}^{k-j} \mathrm{E}\left[\mathrm{e}^{S_{p}} U\left(-S_{p}\right) ; \tilde{M}_{p}<0\right] \mathrm{P}\left(L_{k-j-p} \geq 0\right)
\end{aligned}
$$

Since $U(x)$ is a renewal function, we have $U(x)=O(x), x \rightarrow \infty$. Thus, there exists a constant $C_{3}$ such that $\mathrm{e}^{-x} U(x) \leq u(x):=C_{3} \mathrm{e}^{-x / 2}$ for all $x>0$. Since $\int_{0}^{\infty} u(x) \mathrm{d} x<\infty$, it follows from Lemma 4 and the duality principle that, for every $\varepsilon>0$, there exists a $J_{1} \equiv J_{1}(\varepsilon)<\infty$ such that, for all $k-j>J_{1}$,

$$
\sum_{p=J_{1}}^{k-j} \mathrm{E}\left[\mathrm{e}^{S_{p}} U\left(-S_{p}\right) ; \tilde{M}_{p}<0\right] \mathrm{P}\left(L_{k-j-p} \geq 0\right) \leq \frac{\varepsilon}{2 C_{2}} \mathrm{P}\left(L_{k-j} \geq 0\right) .
$$

Thus, for $k-j>J \geq J_{1}$,

$$
R_{2} \leq \frac{\varepsilon}{2} \mathrm{P}\left(\tilde{M}_{j}<0\right) \mathrm{P}\left(L_{k-j} \geq 0\right)
$$

Now we will evaluate $R_{1}$. For $t<j$, we obtain

$$
\begin{aligned}
\mathrm{E}\left[\mathrm{e}^{S_{\tau(k)}-S_{j}} ; \tau(k)=t\right] & =\mathrm{E}\left[\mathrm{e}^{S_{t}-S_{j}} ; \min _{0 \leq p \leq t-1} S_{p}>S_{t} ; \min _{t \leq p \leq k} S_{p} \geq S_{t}\right] \\
& =\mathrm{E}\left[\mathrm{e}^{S_{t}-S_{j}} ; \min _{t \leq p \leq k} S_{p} \geq S_{t}\right] \mathrm{P}\left(\tilde{M}_{t}<0\right) \\
& =\mathrm{E}\left[\mathrm{e}^{-S_{j-t}} ; \min _{0 \leq p \leq k-t} S_{p} \geq 0\right] \mathrm{P}\left(\tilde{M}_{t}<0\right),
\end{aligned}
$$

where to obtain the second relation we have again used the duality principle. Arguing as before, we see that

$$
\begin{aligned}
& \mathrm{E}\left[\mathrm{e}^{-S_{j-t}} ; \min _{0 \leq p \leq k-t} S_{p} \geq 0\right] \\
& \quad=\mathrm{E}\left[\mathrm{e}^{-S_{j-t}} \mathrm{P}\left(\min _{0 \leq p \leq k-j} S_{p}^{(j-t)} \geq-S_{j-t} \mid S_{j-t}\right) ; \min _{0 \leq p \leq j-t} S_{p} \geq 0\right] \\
& \quad=\mathrm{E}\left[\mathrm{e}^{-S_{j-t}} \lambda_{k-j}\left(S_{j-t}\right) ; L_{j-t} \geq 0\right] \\
& \quad \leq C_{1} \mathrm{P}\left(L_{k-j} \geq 0\right) \mathrm{E}\left[\mathrm{e}^{-S_{j-t}} V\left(S_{j-t}\right) ; L_{j-t} \geq 0\right] .
\end{aligned}
$$

Hence,

$$
\begin{aligned}
R_{1} & \leq C_{1} \mathrm{P}\left(L_{k-j} \geq 0\right) \sum_{t=0}^{j-J} \mathrm{E}\left[\mathrm{e}^{-S_{j-t}} V\left(S_{j-t}\right) ; L_{j-t} \geq 0\right] \mathrm{P}\left(\tilde{M}_{t}<0\right) \\
& =C_{1} \mathrm{P}\left(L_{k-j} \geq 0\right) \sum_{p=J}^{j} \mathrm{E}\left[\mathrm{e}^{-S_{p}} V\left(S_{p}\right) ; L_{p} \geq 0\right] \mathrm{P}\left(\tilde{M}_{j-p}<0\right) .
\end{aligned}
$$


From this bound we can deduce, using Lemma 4 and the same argument as that employed to evaluate $R_{2}$, that for every $\varepsilon>0$ there exists a $J_{2}(\varepsilon)<\infty$ such that, for all $j>J \geq J_{2}$,

$$
R_{1} \leq \frac{\varepsilon}{2} \mathrm{P}\left(\tilde{M}_{j}<0\right) \mathrm{P}\left(L_{k-j} \geq 0\right)
$$

Combining (43) with (44) and setting $J:=\max \left\{J_{1}, J_{2}\right\}$ completes the proof of Lemma 5.

Next we evaluate the contributions to the expectations of interest from the events where $\tau(k)$ is equal to a fixed number close to $j$.

Lemma 6. Under Spitzer's condition, (18), for any fixed $r \in \mathbb{Z}$,

$$
\lim _{j, k-j \rightarrow \infty} \frac{\mathrm{E}\left[\mathrm{e}^{-S_{j}} W_{k}^{-1} ; \tau(k)=j+r\right]}{\mathrm{P}\left(\tilde{M}_{j}<0\right) \mathrm{P}\left(L_{k-j} \geq 0\right)}=\mathrm{E}_{-,+} \frac{\mathrm{e}^{S_{r}^{-}} \mathbf{1}_{\{r \geq 0\}}+\mathrm{e}^{-S_{-r}^{+}} \mathbf{1}_{\{r<0\}}}{\eta_{1}^{-}+\eta_{2}^{+}},
$$

where $\eta_{1}^{-}$and $\eta_{2}^{+}$are independent $R V s$ defined as in (23), but for the independent random walks $\left\{S_{n}^{-}\right\}_{n \geq 0}$ and $\left\{S_{n}^{+}\right\}_{n \geq 0}$, respectively.

Proof. For $0 \leq r \leq k-j$, let

$$
G_{j+r, k-j-r}:=\frac{\mathrm{e}^{S_{r}^{-}}}{\sum_{p=1}^{j+r} \mathrm{e}^{S_{p}^{-}}+\sum_{p=0}^{k-j-r} \mathrm{e}^{-S_{p}^{+}}} .
$$

Then

$$
\begin{aligned}
& \mathrm{E}\left[\mathrm{e}^{-S_{j}} W_{k}^{-1} ; \tau(k)=j+r\right] \\
& \quad=\mathrm{E}\left[\frac{\mathrm{e}^{S_{j+r}-S_{j}}}{\sum_{p=0}^{k} \mathrm{e}^{S_{j+r}-S_{p}}} ; \min _{0 \leq p \leq j+r-1} S_{p}>S_{j+r} ; \min _{j+r \leq p \leq k} S_{p} \geq S_{j+r}\right] \\
& \quad=\mathrm{E}\left[G_{j+r, k-j-r} ; \tilde{M}_{j+r}^{-}<0, L_{k-j-r}^{+} \geq 0\right] \\
& \quad=\mathrm{E}\left[G_{j+r, k-j-r} \mid \tilde{M}_{j+r}^{-}<0, L_{k-j-r}^{+} \geq 0\right] \mathrm{P}\left(\tilde{M}_{j+r}<0\right) \mathrm{P}\left(L_{k-j-r} \geq 0\right) .
\end{aligned}
$$

Clearly, $0<G_{j+r, k-j-r} \leq 1$ and

$$
\lim _{j, k-j \rightarrow \infty} G_{j+r, k-j-r}=\frac{\mathrm{e}^{S_{r}^{-}}}{\eta_{1}^{-}+\eta_{2}^{+}} \quad \mathrm{P}_{-,+} \text {-a.s. }
$$

Hence, by applying Lemma 2 and recalling (25) and the properties of regularly varying functions (cf. (35)), we obtain (45) for $r \geq 0$. The proof of (45) for $r<0$ is almost identical. Lemma 6 is thus proved.

Proof of Theorem 5. For a fixed $\varepsilon>0$, let $J \equiv J(\varepsilon)$ be such that (42) holds. For $j \geq J$ and $n-j \geq J+1$, from (17) we have

$$
\mathrm{E} N_{n}(j)=R_{3}+R_{4}+R_{5},
$$

where

$$
\begin{gathered}
R_{3}:=\sum_{k=j}^{j+J-1} \mathrm{Ee}^{-S_{j}} W_{k}^{-1}, \quad R_{4}:=\sum_{k=j+J}^{n-1} \mathrm{E}\left[\mathrm{e}^{-S_{j}} W_{k}^{-1} ;|\tau(k)-j|<J\right], \\
R_{5}:=\sum_{k=j+J}^{n-1} \mathrm{E}\left[\mathrm{e}^{-S_{j}} W_{k}^{-1} ;|\tau(k)-j| \geq J\right] .
\end{gathered}
$$


We evaluate the quantities $R_{i}, i=3,4,5$, separately. First observe that, in view of (30) (with $n$ replaced by $k$ ), there exists a constant $C_{3}$ such that, for all sufficiently large $j$,

$$
R_{3} \leq C_{3} J \mathrm{P}\left(\tilde{M}_{j}<0\right) .
$$

Thus, since

$$
(n-j) \mathrm{P}\left(L_{n-j} \geq 0\right) \sim(n-j)^{\rho} l_{1}(n-j) \rightarrow \infty \quad \text { as } n-j \rightarrow \infty,
$$

it follows that

$$
R_{3}=o\left((n-j) \mathrm{P}\left(\tilde{M}_{j}<0\right) \mathrm{P}\left(L_{n-j} \geq 0\right)\right) \quad \text { as } n-j \rightarrow \infty .
$$

Furthermore, using the obvious inequality $W_{k} \geq \mathrm{e}^{-S_{\tau(k)}}$ and the bound (42) together with (25) and Karamata's theorem, for $j \geq J$ and some constant $C_{5}>0$ we have

$$
\begin{aligned}
R_{5} & \leq \varepsilon \mathrm{P}\left(\tilde{M}_{j}<0\right) \sum_{k=j+J}^{n-1} \mathrm{P}\left(L_{k-j} \geq 0\right) \\
& =\varepsilon \mathrm{P}\left(\tilde{M}_{j}<0\right) \sum_{p=J}^{n-j-1} \mathrm{P}\left(L_{p} \geq 0\right) \\
& \leq \varepsilon C_{5}(n-j) \mathrm{P}\left(\tilde{M}_{j}<0\right) \mathrm{P}\left(L_{n-j} \geq 0\right)
\end{aligned}
$$

and, therefore,

$$
\frac{R_{5}}{(n-j) \mathrm{P}\left(\tilde{M}_{j}<0\right) \mathrm{P}\left(L_{k-j} \geq 0\right)} \leq \varepsilon C_{5} .
$$

Finally, set

$$
E_{J}:=\mathrm{E}_{-,+} \frac{1+\sum_{r=1}^{J-1}\left(\mathrm{e}^{S_{r}^{-}}+\mathrm{e}^{-S_{r}^{+}}\right)}{\eta_{1}^{-}+\eta_{2}^{+}} .
$$

Using Lemma 6, (25), and the properties of regularly varying functions, we see that, as $\min \{j, n-j\} \rightarrow \infty$,

$$
\begin{aligned}
R_{4} & \sim E_{J} \mathrm{P}\left(\tilde{M}_{j}<0\right) \sum_{k=j+J}^{n-1} \mathrm{P}\left(L_{k-j} \geq 0\right) \\
& \sim E_{J} \mathrm{P}\left(\tilde{M}_{j}<0\right) \sum_{p=J}^{n-j-1} \mathrm{P}\left(L_{p} \geq 0\right) \\
& \sim E_{J} \mathrm{P}\left(\tilde{M}_{j}<0\right) \rho^{-1}(n-j) \mathrm{P}\left(L_{n-j} \geq 0\right) .
\end{aligned}
$$

Since $\lim _{J \rightarrow \infty} E_{J}=1$ by the dominated convergence theorem, the assertion of Theorem 5 immediately follows from the above relation for $R_{4}$ and the bounds for $R_{3}$ and $R_{5}$.

\subsection{The asymptotic behaviour of the distribution of $\mathrm{E}_{w} N_{n}(j)$}

Unfortunately, our description of the asymptotic behaviour of $\mathrm{E}_{w} N_{n}(j)$ will be less detailed than that for $\mathrm{E} N_{n}(j)$. We will be able to describe the distribution of the $\mathrm{RV}_{w} N_{n}(j)$ only for values of $j$ located either to the right or in a small left-hand vicinity of the random epoch $\tau(n)$. 
Theorem 6. Let Spitzer's condition, (18), be satisfied and let $j \equiv j(n)$ be an arbitrary (random) sequence with the property that $(\tau(n)-j)_{+}=o(n)$ in probability as $n \rightarrow \infty$. Then

$$
\mathrm{P}\left(\frac{\mathrm{e}^{S_{j}-S_{\tau(n)}}}{n-j} \mathrm{E}_{w} N_{n}(j)<x\right) \Rightarrow \mathrm{P}_{-,+}\left(\frac{1}{\eta_{1}^{-}+\eta_{2}^{+}}<x\right),
$$

where $\eta_{1}^{-}$and $\eta_{2}^{+}$are RVs defined as in (23), but for the independent random walks $\left\{S_{n}^{-}\right\}_{n \geq 0}$ and $\left\{S_{n}^{+}\right\}_{n \geq 0}$, respectively.

Proof. Since the RVs $W_{n}$ (see (5)) are increasing in $n$, from (16) we have the following lower bound:

$$
\mathrm{E}_{w} N_{n}(j) \geq(n-j) \mathrm{e}^{-S_{j}} W_{n}^{-1}=\frac{(n-j) \mathrm{e}^{S_{\tau(n)}-S_{j}}}{\sum_{k=0}^{n} \mathrm{e}^{S_{\tau(n)}-S_{k}}} .
$$

Now we will derive an upper bound for $\mathrm{E}_{w} N_{n}(j)$. To this end observe that, according to (23), for any fixed $\varepsilon>0$ and $\delta>0$ there exists a $J<\infty$ such that

$$
\mathrm{P}^{+}\left(\sum_{k=J}^{\infty} \mathrm{e}^{-S_{k}}>\delta\right) \leq \varepsilon
$$

Clearly, for any $j \in[\tau(n), n-1]$,

$$
\begin{aligned}
\mathrm{E}_{w} N_{n}(j) & \leq \mathrm{e}^{S_{\tau(n)}-S_{j}}(\tau(n)+J-j)_{+}+\mathrm{e}^{-S_{j}}(n-j) W_{\tau(n)+J}^{-1} \\
& =\mathrm{e}^{S_{\tau(n)}-S_{j}}\left[(\tau(n)+J-j)_{+}+(n-j)\left(\sum_{k=0}^{\tau(n)+J} \mathrm{e}^{S_{\tau(n)}-S_{k}}\right)^{-1}\right] .
\end{aligned}
$$

Hence, we obtain

$$
\begin{aligned}
\left(\sum_{k=0}^{n} \mathrm{e}^{S_{\tau(n)}-S_{k}}\right)^{-1} & \leq \frac{\mathrm{e}^{S_{j}-S_{\tau(n)}}}{n-j} \mathrm{E}_{w} N_{n}(j) \\
& \leq \frac{(\tau(n)+J-j)_{+}}{n-j}+\left(\sum_{k=0}^{\tau(n)+J} \mathrm{e}^{S_{\tau(n)}-S_{k}}\right)^{-1} .
\end{aligned}
$$

Evidently, for $y>0$,

$$
\begin{aligned}
\mathrm{P}\left(\sum_{k=0}^{n} \mathrm{e}^{S_{\tau(n)}-S_{k}}<y\right) & =\sum_{p=0}^{n} \mathrm{P}\left(\sum_{k=0}^{n} \mathrm{e}^{S_{\tau(n)}-S_{k}}<y ; \tau(n)=p\right) \\
& =\sum_{p=0}^{n} \mathrm{P}\left(\sum_{l=1}^{p} \mathrm{e}^{S_{l}^{-}}+\sum_{r=0}^{n-p} \mathrm{e}^{-S_{r}^{+}}<y ; \tilde{M}_{p}^{-}<0, L_{n-p}^{+} \geq 0\right) .
\end{aligned}
$$

Furthermore, from (23) and (24) note that, as $\min \{p, n-p\} \rightarrow \infty$,

$$
\mathrm{P}\left(\sum_{l=1}^{p} \mathrm{e}^{S_{l}^{-}}+\sum_{r=0}^{n-p} \mathrm{e}^{-S_{r}^{+}}<y \mid \tilde{M}_{p}^{-}<0, L_{n-p}^{+} \geq 0\right) \Rightarrow \mathrm{P}_{-,+}\left(\eta_{1}^{-}+\eta_{2}^{+}<y\right) .
$$


If condition (18) is met, then the generalized arcsine law holds (see, e.g. Theorems 8.9.9 and 8.9.5 of [7]):

$$
\lim _{n \rightarrow \infty} \mathrm{P}\left(\frac{\tau(n)}{n} \leq x\right)=\frac{\sin \pi \rho}{\pi} \int_{0}^{x} t^{\rho-1}(1-t)^{-\rho} \mathrm{d} t, \quad x \in[0,1] .
$$

Thus, for any $\varepsilon_{1}>0$, there exists a $\delta_{1} \in\left(0, \frac{1}{2}\right)$ such that

$$
\mathrm{P}\left(\tau(n) \notin\left(n \delta_{1}, n\left(1-\delta_{1}\right)\right)\right) \leq \varepsilon_{1},
$$

which, combined with (49) and (50), shows that, as $n \rightarrow \infty$,

$$
\mathrm{P}\left(\sum_{k=0}^{n} \mathrm{e}^{S_{\tau(n)}-S_{k}}<y\right) \Rightarrow \mathrm{P}_{-,+}\left(\eta_{1}^{-}+\eta_{2}^{+}<y\right) .
$$

A similar argument combined with (47) shows that

$$
\mathrm{P}\left(\sum_{k=0}^{\tau(n)+J} \mathrm{e}^{S_{\tau(n)}-S_{k}}<y\right) \Rightarrow \mathrm{P}_{-,+}\left(\eta_{1}^{-}+\eta_{2}^{+}<y\right)
$$

as first $n \rightarrow \infty$ and then $J \rightarrow \infty$. On the other hand, again using (51), we conclude that, for $j \in[\tau(n), n-1]$,

$$
\begin{aligned}
\frac{(\tau(n)+J-j)_{+}}{n-j} \leq & \mathbf{1}_{\{\tau(n)+J>j\}} \frac{J}{n-j} \mathbf{1}_{\{\tau(n) \geq n-\sqrt{n}\}} \\
& \quad+\mathbf{1}_{\{\tau(n)+J>j\}} \frac{J}{\sqrt{n}-J} \mathbf{1}_{\{\tau(n)<n-\sqrt{n}\}} \\
& \leq J \mathbf{1}_{\{\tau(n) \geq n-\sqrt{n}\}}+\frac{J}{\sqrt{n}-J} \\
& \stackrel{\mathrm{P}}{\rightarrow} 0
\end{aligned}
$$

as first $n \rightarrow \infty$ and then $J \rightarrow \infty$.

Using (53) on the left-hand side of (48), and (54) and (56) on the right-hand side of (48), proves (46) for $j \in[\tau(n), n-1]$.

For $\tau(n)-j>0$, we can use similar arguments. The only difference is that, in this case,

$$
(\tau(n)+J-j)_{+}=\tau(n)+J-j,
$$

and for $j<\tau(n)$ (varying with $n$ in such a way that $(\tau(n)-j)_{+}=o(n)$ ) the conclusion (56) still holds, by (52). Theorem 6 is thus proved.

\section{Acknowledgements}

This research was supported by the ARC Centre of Excellence for Mathematics and Statistics of Complex Systems. The second author was also supported by the Russian Foundation for Basic Research (grant 05-01-00035) and by the program 'Contemporary Problems of Theoretical Mathematics' of the Russian Academy of Sciences. He is grateful to the Department of Mathematics and Statistics of the University of Melbourne for its hospitality during his visit there.

The authors are very grateful to the referee for his valuable comments, which improved the exposition of the paper. 


\section{References}

[1] Afanasyev, V. I., Geiger, J., Kersting, G. and Vatutin, V. A. (2005). Criticality for branching processes in random environment. Ann. Prob. 33, 645-673.

[2] Babilot, M., Bougerol, P. and Élie, L. (1997). The random difference equation $X_{n}=A_{n} X_{n-1}+B_{n}$ in the critical case. Ann. Prob. 25, 478-493.

[3] Barabási, A. L. ANd Albert, R. (1999). Emergence of scaling in random networks. Science 286, 509-512.

[4] Bertoin, J. And Doney, R. A. (1994). On conditioning a random walk to stay nonnegative. Ann. Prob. 22, 2152-2167.

[5] Biggins, J. D. And Grey, D. R. (1997). A note on the growth of random trees. Statist. Prob. Lett. 32, 339-342.

[6] Billingsley, P. (1968). Convergence of Probability Measures. John Wiley, New York.

[7] Bingham, N. H., Goldie, C. M. and Teugels, J. L. (1987). Regular Variation. Cambridge University Press.

[8] Bollobás, B., Riordan, O., Spencer, J. and Tusnády, G. (2001). The degree of a scale-free random graph process. Random Structures Algorithms 18, 279-290.

[9] Borovkov, A. A. (1976). Stochastic Processes in Queueing Theory. Springer, New York.

[10] Borovkov, K. A. AND Motyer, A. (2005). On the asymptotic behavior of a simple growing point process model. Statist. Prob. Lett. 72, 265-275.

[11] Devroye, L. (1988). Applications of the theory of records in the study of random trees. Acta Informatica 26, 123-130.

[12] Doney, R. A. (1995). Spitzer's condition and ladder variables in random walks. Prob. Theory Relat. Fields 101, $577-580$.

[13] Élie, L. (1982). Compartement asymptotic du noyau potentiel sur les groupes de Lie. Ann. Sci. École Norm. Sup. 15, 257-364.

[14] Hirano, K. (1998). Determination of the limiting coefficient for exponential functionals of random walks with positive drift. J. Math. Sci. Univ. Tokyo 5, 299-332.

[15] Mahmoud, H. (1992). Evolution of Random Search Trees. John Wiley, New York.

[16] Pittel, B. (1994). Note on the height of recursive trees and random $m$-ary search trees. Random Structures Algorithms 5, 337-347.

[17] Rüschendorf, L. And Rachev, S. (1995). Probability metrics and recursive algorithms. Adv. Appl. Prob. 27, 770-799.

[18] Spitzer, F. (1964). Principles of Random Walk. Van Nostrand, Princeton, NJ.

[19] Vatutin, V. A. And Dyakonova, E. E. (2004). Galton-Watson branching processes in random environment. II. Finite-dimensional distributions. Theory Prob. Appl. 49, 275-308. 